\title{
Diseño de un robot móvil controlado por un agente reactivo en tiempo real
}

\author{
Griselda Saldaña ${ }^{1}$, Jorge Cerezo ${ }^{1}$, Mario Mauricio Bustillo ${ }^{2}$, Apolonio Ata ${ }^{2}$, \\ Beatriz Bernabé ${ }^{2}$, Gerardo Martínez ${ }^{2}$, Rogelio González ${ }^{2}$ \\ ${ }^{1}$ Universidad Tecnológica de Puebla, \\ Ingeniería en Tecnologías para la Automatización, México \\ ${ }^{2}$ Benemérita Universidad Autónoma de Puebla, \\ Facultad de Ciencias de la Computación, México \\ \{griselda.saldana, jorge.cerezo\} @utpuebla.edu.mx, \\ bustillo1956@hotmail.com, \\ \{apolonio.ata, beatriz.bernabe\}@gmail.com, \{gguzman, \\ rgonzalez\}@es.buap.mx
}

Resumen. En este trabajo se presenta el desarrollo de un sistema de detección de objetos en un espacio bidimensional controlado, empleando técnicas de visión artificial. Los objetos detectados tienen una geometría rígida y están expuestos a iluminación real, por lo tanto, el sistema es robusto a los cambios de iluminación y sombreado. Con el fin de manejar la gran cantidad de datos a procesar en tiempo real, se utiliza un dispositivo MyRIO el cual contiene un FPGA. Dicho dispositivo permite la comunicación con el software LabVIEW donde reside la interfaz de usuario. Empleando LabVIEW se implementa un algoritmo de seguimiento por color, con el fin de asistir a un agente reactivo, que utiliza un sensor infrarrojo para detectar la distancia a un obstáculo y realizar las funciones de forrajeo y almacenamiento.

Palabras clave: Seguimiento de objetos, LabVIEW, detección por color, robot móvil.

\section{Design of a Mobile Robot Controlled by a Real-Time Agent}

\begin{abstract}
In this paper the development of an object detection system in a controlled two-dimensional space using computer vision techniques is presented. The detected objects have a rigid geometry and are exposed to real light; therefore, the system is robust to changes in lighting and shading. In order to handle the large amount of data to be processed in real time, a MyRIO device which contains an FPGA is used. This device allows communication with the
\end{abstract}


LabVIEW software where the user interface resides. Using LabVIEW a tracking by color algorithm is implemented, in order to attend to a reactive agent, which uses an infrared sensor to detect the distance to an obstacle and perform the functions of foraging and storage.

Keywords. Tracking, LabVIEW, color detection, mobile robot.

\section{Introducción}

Actualmente la visión juega un papel central en el área de robótica para una serie de tareas como la auto-localización, la navegación, el reconocimiento y manipulación de objetos, el seguimiento de objetivos, la interacción social entre humano y robot, imitación, entre otros [1]. En los últimos años, los robots móviles han participado en tareas más y más complejas que a menudo requieren la colaboración entre varios individuos que, en general, difieren en sus habilidades y en su forma de percibir el entorno externo [2]. Para llevar a cabo dichas tareas, los robots móviles suelen estar equipados con potentes sistemas de visión, donde la operación en tiempo real se convierte en un desafío. Por otra parte, cuando se considera un sistema de múltiples robots, el control y la coordinación son tareas complejas y demandantes.

De acuerdo con [3] los sistemas de visión para agentes móviles se pueden clasificar en seis tipos, sensado y precepción [4-8]; mapeo y auto-localización $[9,10,11]$; reconocimiento y localización $[12,13,14,15,16]$; navegación y planeación $[17,18]$; seguimiento [19, 20, 21]; y control por visión (servoing) [22, 23, 24].

En este trabajo, se desarrolla un sistema de visión montado en un robot móvil, el cual es capaz de navegar en un ambiente controlado empleando una cámara. El sistema está inspirado en el fenómeno de división de tareas, que se lleva a cabo en algunas colonias de insectos para realizar una tarea compleja común a un grupo como es el caso del forrajeo [25]. La tarea del robot consiste en observar su entorno para identificar un objeto de interés de un color específico, navegar hacia él y asirlo para transportarlo a un área de almacenaje. El agente controla de forma independiente una cámara de manera que puede dirigir su atención a diferentes objetos del entorno. Por otro lado, el robot cuenta con sus propios recursos de cómputo que le permiten analizar la imagen al emplear un algoritmo de seguimiento en base a color. Para procesar las imágenes en tiempo real se utiliza una tarjeta MyRIO 1900 que incluye un FPGA, el cual permite procesamiento en paralelo. El robot cuenta además con los recursos necesarios para su comunicación con otros agentes vía una red Wi-Fi. El sistema se programó empleando LabVIEW, que es un entorno de desarrollo para medición y automatización, desarrollado por National Instruments (NI) [26, 27], el cual cuenta con un conjunto de herramientas de Visión [28] y de gestión de gráficos e interfaces de usuario.

El documento está organizado de la siguiente manera, en la sección 2 se presenta una descripción del sistema, destacando la construcción del agente así como su programación en LabVIEW, en la sección 3 se muestran las pruebas realizadas al sistema así como algunos resultados. Finalmente, en la sección 4 se presentan las conclusiones y trabajo futuro. 


\section{Descripción del sistema}

La configuración del sistema está compuesta por un robot móvil tipo VEX Clawbot [29], el cual tiene montada en su estructura una tarjeta MyRIO que se comunica de forma inalámbrica con una computadora central donde reside la interface de usuario. En dicha tarjeta se encuentra conectada una cámara de alta definición montada en un servomotor que le permite movimiento en la dirección horizontal (paneo). El robot cuenta además con dos servomotores para su locomoción y uno más para apertura/cierre de la pinza. En la parte frontal se encuentra un sensor infrarrojo que le permite determinar la distancia a los objetos de interés.

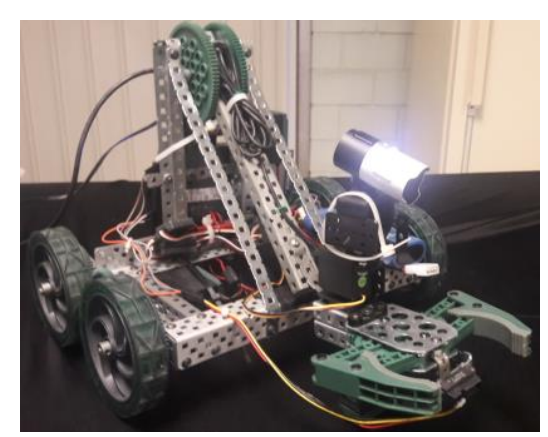

Fig. 1. Prototipo del Robot Móvil.

La tarjeta MyRIO cuenta con un Router Inalámbrico que gestiona las comunicaciones vía WiFi, utilizando variables globales mapeadas a la red que permiten utilizar la información disponible. Adicionalmente esta tarjeta permite la operación STAND-ALONE del sistema ya que el programa desarrollado se ejecuta dentro de los módulos de la misma.

\subsection{Seguimiento en base a color}

Para realizar el seguimiento de los objetos detectados, las imágenes obtenidas se convierten al formato HSI y a continuación se determina la escala para filtrado del color deseado, verde en este caso.

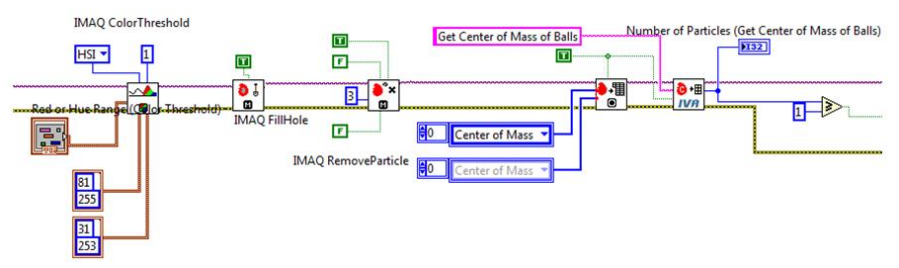

Fig. 2. Procesamiento de la Imagen para seguimiento por color. 
Dentro de los límites de la imagen, el sistema de visión discrimina toda la gama de colores que no correspondan con el filtro programado (se elimina el fondo), dejando visibles solamente los elementos de color especificados. En la figura 3 se observan dos pelotas detectadas, donde se discrimina el resto de la imagen.

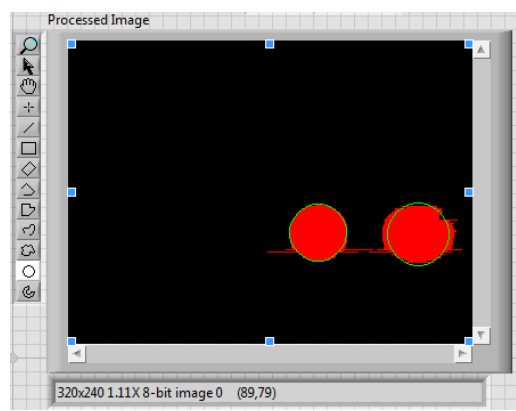

Fig. 3. Seguimiento de objetos de color verde, (el segundo en movimiento).

\subsection{Interface gráfica de usuario}

La interface desarrollada cuenta con un alto grado de usabilidad y funcionalidad. Cuenta con elementos que permiten configurar los parámetros para la máquina de visión, el ancho de pulso para los servomotores del robot, permite modificar en tiempo de ejecución, seleccionar la búsqueda de otra gama de color, así como visualizar la adquisición en tiempo real de la imagen obtenida por la cámara y la imagen procesada. Es posible observar el número de objetos detectados, así como las coordenadas $(x, y)$ de sus centros de masa. También cuenta con dos indicadores de la actividad del agente, uno para el punto en que el robot se encuentra alineado con el objeto de interés (en rango) y otro para el momento en que la pinza puede tomar el objeto (pre-ajuste). La figura 4 muestra el aspecto de la interface de usuario completa.

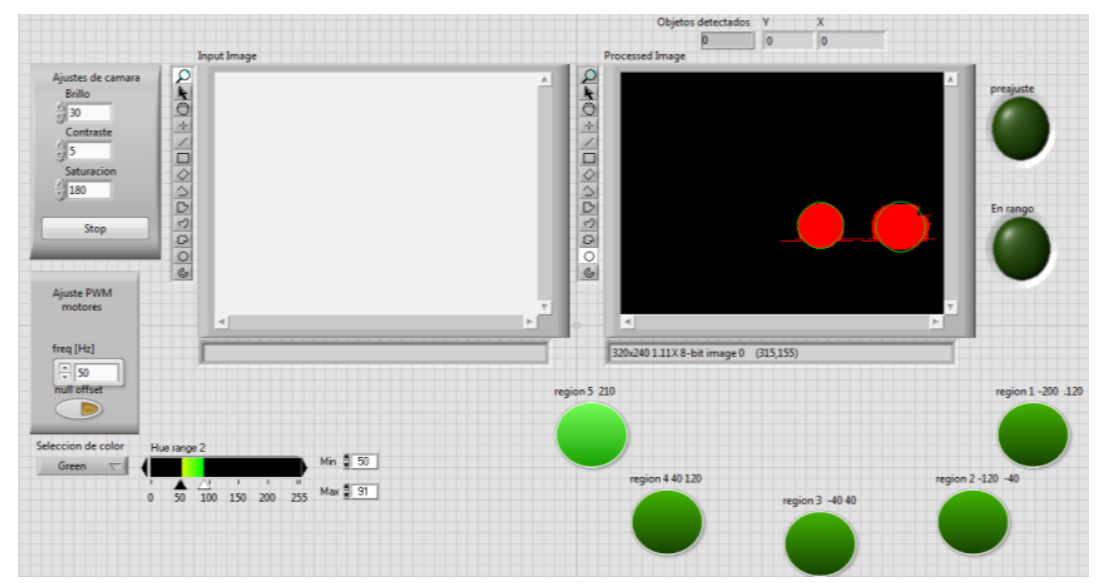

Fig. 4. Interface de usuario. 


\subsection{Diseño del agente reactivo}

Para que el robot pueda interactuar con el entorno, se diseñó una máquina de estados que se inspira en un agente reactivo [30], que da solución a un problema local, dentro de una cancha de pruebas con un área específica de $1.68 \times 2.24$ metros bajo un ambiente de luz real; se considera además un rango de visión para la cámara de 0 a 180 grados. El sistema está diseñado modularmente para agregar nuevas acciones, patrones de comportamiento o agentes adicionales.

Durante la percepción, la cámara realiza un proceso de paneo, que es interrumpido cuando existe un objeto de interés en la pantalla y el centro del mismo se encuentra alineado con el eje $X$ de la cámara.

Una vez localizado el centro del objeto, el plan programado para el agente consiste en determinar en cuál de las 5 zonas de visión posibles en que se ha dividido la imagen se encuentra el objeto. A continuación, el agente define las acciones de control para que el móvil alinee la cámara con el objeto a sujetar.

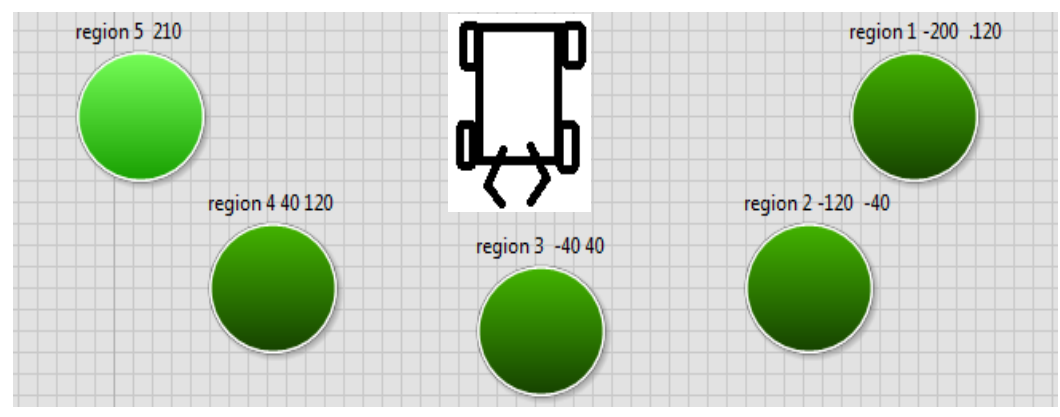

Fig. 5. Regiones del campo de visión del robot en grados.

Cuando la cámara montada en el robot se encuentra en la zona central (zona 3), se procede a realizar un movimiento fino de ajuste para centrar el objeto con la pinza y determinar qué tan lejos se encuentra el móvil del objeto de interés empleando el sensor infrarrojo para finalmente avanzar y sujetarlo.

Es importante recalcar que si por efectos de la mecánica de locomoción del robot o movimiento del objeto de interés, el móvil y la pelota se desalinean, el sistema de percepción del agente permite corregir en tiempo de ejecución la posición del robot y realizar nuevamente un paneo y acciones de alineación en dependencia de los cambios del entorno.

En la figura 6 se muestra la implementación del agente en una máquina de estados desarrollada en LabVIEW.

Este comportamiento realizado por el agente, corresponde a la función de forrajeo, ya que al asir el objeto de interés, el robot regresa a un punto de origen, donde recopila los objetos para que posteriormente otro agente se encargue de llevar a cabo el almacenamiento. 


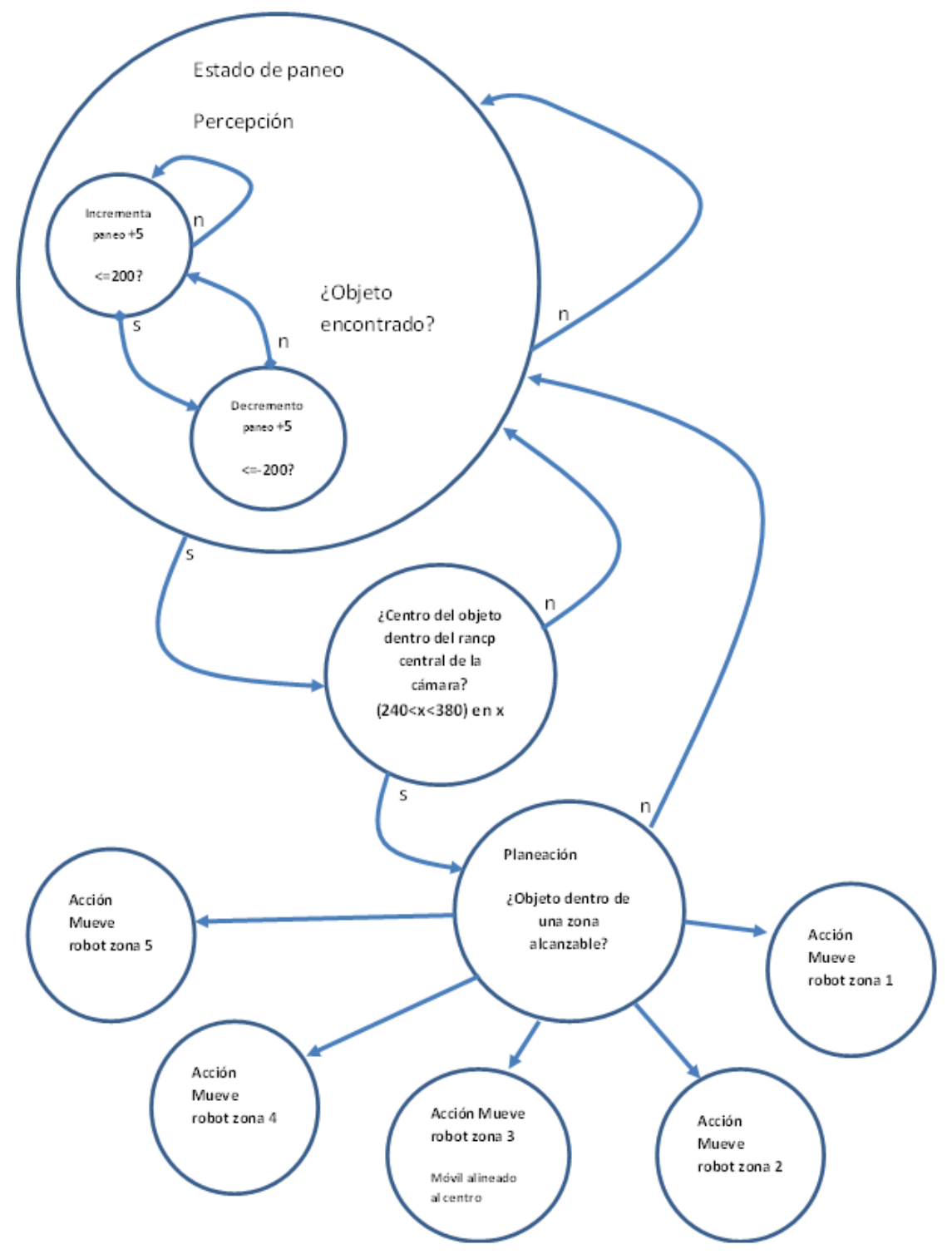

Fig. 6. Diseño del agente reactivo para el sistema de visión.

\section{Pruebas y resultados}

Se realizaron múltiples experimentos considerando el espacio que abarca la cancha de pruebas. 


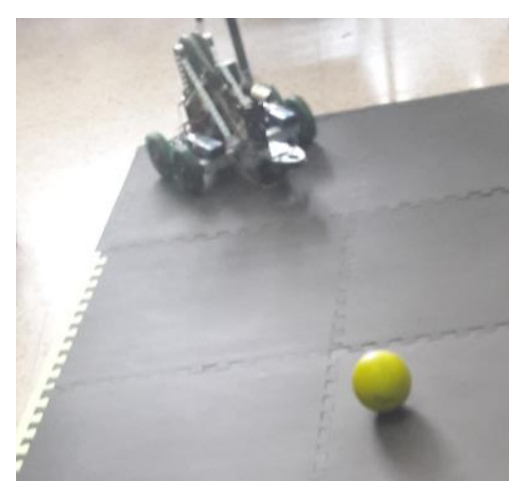

Fig. 7. Cancha de pruebas.

En este espacio se colocaron el robot y una pelota de color verde, y se verificó que desde cualquier punto el robot pudiera identificar la pelota, alinearse, tomarla y dejarla en una posición especificada. El sistema se probó bajo luz artificial y se comprobó que es robusto ante ruido y cambios de iluminación y sombreado.

Para el correcto funcionamiento del sistema, un paso muy importante fue la calibración del color, ya que dependiendo de los pesos que se dan a la saturación y la tonalidad (HSI) la discriminación puede o no mejorar. El valor de la luminosidad hace que el sistema sea más o menos inmune a los cambios de luz del ambiente.

Previamente a la utilización de este algoritmo, se probaron, Traditional mean shift y Shape adapted mean, en ambos casos se intentó detectar 4 pelotas de color verde tomando como parámetros Brillo: 30, Contraste: 5, Saturación: 180 y se obtuvieron los resultados mostrados en la Tabla 1.

Tabla 1. Objetos detectados por algoritmo.

\begin{tabular}{lcc}
\hline Algoritmo & $\begin{array}{c}\text { Objetos detectados con } \\
\text { luz controlada }\end{array}$ & $\begin{array}{c}\text { Objetos detectados } \\
\text { bajo condiciones } \\
\text { variables de luz }\end{array}$ \\
\hline \hline Traditional mean shift & 3 a 4 pelotas & 0 a 3 pelotas \\
Shape adapted mean & 3 a 4 pelotas & 0 a 3 pelotas \\
Basado en calibración de color & 4 pelotas & 4 pelotas \\
\hline
\end{tabular}

Para cada algoritmo se realizaron aproximadamente 50 pruebas con condiciones de luz controladas y a diferentes distancias del robot, obteniendo un $97 \%$ de detección para el algoritmo propuesto. Para el caso en que las condiciones de luz son variables, también se realizaron 50 pruebas para cada algoritmo y en este caso se obtuvo un $93 \%$ de detección con el algoritmo propuesto, que supera a los probados anteriormente cuyo porcentaje varía entre $86 \%$ y $90 \%$ de detección.

Para hacer que el robot se alineara con el objeto de interés, con la finalidad de asirlo, se decidió dividir el campo de visión de la cámara de paneo en 5 zonas, para proceder a hacer un ajuste fino de la pinza. Esto como resultado de múltiples pruebas 
de los movimientos del robot para lograr que reaccionara a modificaciones del entorno.

Para conseguir que el robot pudiera asir los objetos de interés, se realizó un ajuste "fino" de la pinza por lo que fue necesario regular la velocidad de avance del robot, manipulando los anchos de pulso para los motores.

Con la misma finalidad, se calibró el telémetro (infrarrojo) para ajustarlo a una medida en unidades del mundo real.

A pesar de que el sistema es robusto, aún no se ha solucionado el caso en que exista oclusión de los objetos. Cada pelota dentro del sistema de visión recibe una etiqueta. Si dos pelotas dentro del área de visión del robot se cruzan, puede ocurrir que las etiquetas se inviertan. Si este caso se presenta, el robot aun será capaz de asir alguna de las dos y para el comportamiento deseado no es relevante cual tome primero. Por otro lado si dos pelotas se ubican una al lado de la otra el sistema de visión puede reconocerlas como un solo objeto, lo que no afecta en la alineación del robot, pero si en el momento de sujetar el objeto. Si algún objeto se ubica detrás del robot, el sistema de visión continuaría de manera indefinida en el estado de paneo.

\section{Conclusiones}

En este trabajo se ha demostrado que es posible utilizar un robot que pueda realizar una tarea común a un grupo tal como lo es el forrajeo. El uso del entorno LabVIEW permitió reducir el tiempo de implementación y realizar el análisis en tiempo real del sistema de visión y del agente. El agente implementado ejecuta las acciones de control del robot y es capaz de hacer correcciones en tiempo de ejecución. El sistema implementado es modular y configurable. Finalmente, a diferencia de otros trabajos similares, este sistema tiene la ventaja de que permite la comunicación entre agentes o hacia otros dispositivos a través de Wi-Fi gracias a la utilización del dispositivo MyRIO.

Como trabajo futuro se propone implementar un segundo robot que se encargue de la función de almacenamiento y hacer pruebas de comunicación entre agentes. Por otro lado, se implementará un algoritmo de evasión de obstáculos que permita realizar una tarea de mayor complejidad común al conjunto de agentes. Finalmente, para eliminar los problemas de oclusión, se utilizará un dispositivo Kinect que proporcione una vista superior del área de pruebas para identificar en todo momento la posición de los objetos de interés incluyendo el caso en que dichos objetos se encuentren detrás del robot.

Agradecimientos. Los autores agradecen al PRODEP por el apoyo para la realización de este trabajo.

\section{Referencias}

1. Fiack, L., Cuperlier, N., Miramond, B. J.: Embedded and real-time architecture for bioinspired vision-based robot navigation. Journal of Real-Time Image Processing, 10 (4), 699-722 (2015) 
2. Infantino, I., Cossentino, M., Chella, A.: An Agent Based Multilevel Architecture for robotics vision systems. Proceedings of the International Conference on Artificial Intelligence, 386-390 (2002)

3. Martínez-Gómez, J. et. al.: A taxonomy of Vision Systems for Ground Mobile Robots. International Journal of Advanced Robotic Systems, 11 (7) (2004)

4. Zhao, Z., Weng., Y.: A flexible method combining camera calibration and hand-eye calibration. Robótica, 31 (5), 747-756 (2013)

5. Peynot, T., Scheding, S. and Terho, S.: The marulan data sets: Multi-sensor perception in a natural environment with challenging conditions. International Journal of Robotics Research, 29 (13), 1602-1607 (2010)

6. Fernández-Caballero, A., Castillo, J. C., Martínez-Cantos, J., Martínez-Tomás, R.: Optical flow or image subtraction in human detection from infrared camera on mobile robot. Robotics and Autonomous Systems, 58 (12), 1273-1281 (2010)

7. Ekmanis, M., Nikitenko, A.: Mobile Robot Camera Extrinsic Parameters Auto Calibration by Spiral Motion. Engineering for Rural Development Jelgava, 558-565 (2016)

8. Di Paola, D., et al.: Multi-sensor surveillance of indoor environments by an autonomous mobile robot. Int. J. Intelligent Systems Technologies and Applications, 8, $18-35$ (2010)

9. Ahmmed, P., et al.: Self-localization of a mobile robot using monocular vision of a chessboard pattern. International Conference on Electrical and Computer Engineering, (2014)

10. Biswas, J., Veloso, M.: Depth camera based indoor mobile robot localization and navigation. International Conference on Robotics and Automation, 1697-1702 (2012)

11. Çelik, K., Somani, A. K.: Monocular vision SLAM for indoor aerial vehicles. Journal of Electrical and Computer Engineering (2013)

12. Cocias, T. T., Moldoveanu, F., Grigorescu, S. M.: Generic fitted shapes (gfs): Volumetric object segmentation in service robotics. Robotics and Autonomous Systems, 61 (9), 960-972 (2013)

13. Xiao, J., Adler, B., Zhang, J., Zhang, H.: Planar segment based three-dimensional point cloud registration in outdoor environments. Journal of Field Robotics, 30 (4), 552-582 (2013)

14. Yeom S., Woo, Y.-H.: Person-specific face detection in a scene with optimum composite filtering and colour-shape information. International Journal of Advanced Robotic Systems, 10 (70) (2013)

15. Zhang, J., Zhang, J., Chen, S.: Discover novel visual categories from dynamic hierarchies using multimodal attributes. IEEE Transactions on Industrial Informatics, 9 (3), 1688-1696 (2013)

16. Kawasue, K., Komatsu, T.: Shape measurement of a sewer pipe using a mobile robot with computer vision. International Journal of Advanced Robotic Systems, 10 (52) (2013)

17. Kang, T. K., Choi, I.-H., Park, G.-T., Lim, M.-T.: Local environment recognition system using modified surf-based $3 \mathrm{~d}$ panoramic environment map for obstacle avoidance of a humanoid robot. International Journal of Advanced Robotic Systems, 10 (275) (2013)

18. Papadakis, P.: Terrain traversability analysis methods for unmanned ground vehicles: A survey. Engineering Applications of Artificial Intelligence, 26 (4), 1373-1385 (2013) 
19. Park, J., Hwang, W., Kwon, H., Kim, K., Cho, D.-I.D.: A novel line of sight control system for a robot vision tracking system, using vision feedback and motiondisturbance feedforward compensation. Robotica, 31 (1), 99-112 (2013)

20. Ostafew, C. J., Schoellig, A. P., Barfoot, T. D.: Learning-Based Nonlinear Model Predictive Control to Improve Vision-Based Mobile Robot Path-Tracking in Challenging Outdoor Environments. International Conference on Robotics \& Automation, 4029-4036 (2014)

21. Basso, F., Munaro, M., Michieletto, S., Pagello, E., Menegatti, E.: Fast and robust multi-people tracking from RGB-D data for a mobile robot. Intelligent Autonomous Systems, 265-276 (2013)

22. Siradjuddin, I., Behera, L., McGinnity, T. M., Coleman, S.: Image-Based Visual Servoing of a 7-DOF Robot Manipulator Using an Adaptive Distributed Fuzzy PD Controller. IEEE/ASME Transactions on Mechatronics, 19 (2) (2014)

23. Fomena, R. T., et al.: Coarsely Calibrated Visual Serving of a Mobile Robot using a Catadioptric Vision System. IEEE/RSJ International Conference on Intelligent Robots and Systems, 5432-5437 (2009)

24. Wang, K., Liu, Y., Li, L.: Visual Servoing Trajectory Tracking of Nonholonomic Mobile Robots Without Direct Position Measurement. IEEE Transactions on Robotics, 30 (4), 1026-1035 (2014)

25. Dorigo, M., Birattari, M. and Stützle, T.: Ant Colony Optimization: Artificial Ants as a Computational Intelligence Technique. IRIDIA - Technical Report Series: TR/IRIDIA/2006-023, 1-12 (2006)

26. Moreno Astorga, A., Moreno-Salinas, D., Chaos García, D., Aranda Almansa, J.: Simulation benchmark for autonomous marine vehicles in LabView. In: Proceeding of OCEANS Conference, 1-6 (2011)

27. De Asmundis R.: The Importance of a Deep Knowledge of LabVIEW Environment and Techniques in Order to Develop Effective Applications. Practical Applications and Solutions Using LabVIEW ${ }^{\mathrm{TM}}$ Software, Dr. Silviu Folea (Ed.), InTech (2011)

28. Relf, C. G.: Image Acquisition and Processing with LabVIEW. CRC Press (2004)

29. VEX Robotics, http://www. vexrobotics.com.mx/

30. Brooks, R. A.: A Robust Layered Control System for a Mobile Robot. IEEE Journal on Robotics and Automation. 2 (1), 14-23(1986) 